\title{
Reducing the risk of hypocalcaemia with parenteral antiresorptive therapies: an audit
}

Wei Xu, Kenneth Baker, Emily Oates, Rachel Reaveley, Terry Aspray

Metabolic Bone Service, Freeman Hospital, Newcastle upon Tyne, UK

\section{Introduction}

Intravenous bisphosphonates and subcutaneous denosumab are potent antiresorptive agents widely used in the treatment of osteoporosis, Paget's disease and metastatic malignancy (1). Several case reports have identified the risk of lifethreatening hypocalcaemia with these treatments (2-4), as highlighted by recent UKMHRA advice (5). Vitamin D deficiency contributes to this risk and it is recommended that supplements are given where necessary.

\section{Local guidlines}

- All patients should be vitamin D replete (serum $(25 \mathrm{OH})$ vitamin D > 50nmol/L)

Measurement interval depends on drug:

Zoledronate \& denosumab - within past 2 months of infusion.

Other bisphosphonates - within past 12 months

- If $25 \mathrm{OHD}<50 \mathrm{nmol} / \mathrm{L}$ - defer infusion and give vitamin $\mathrm{D}$ replacement in the form of Dekristol 100000 units (can all be taken at once, or staggered if preferred).

\section{Result of first audit cycle}

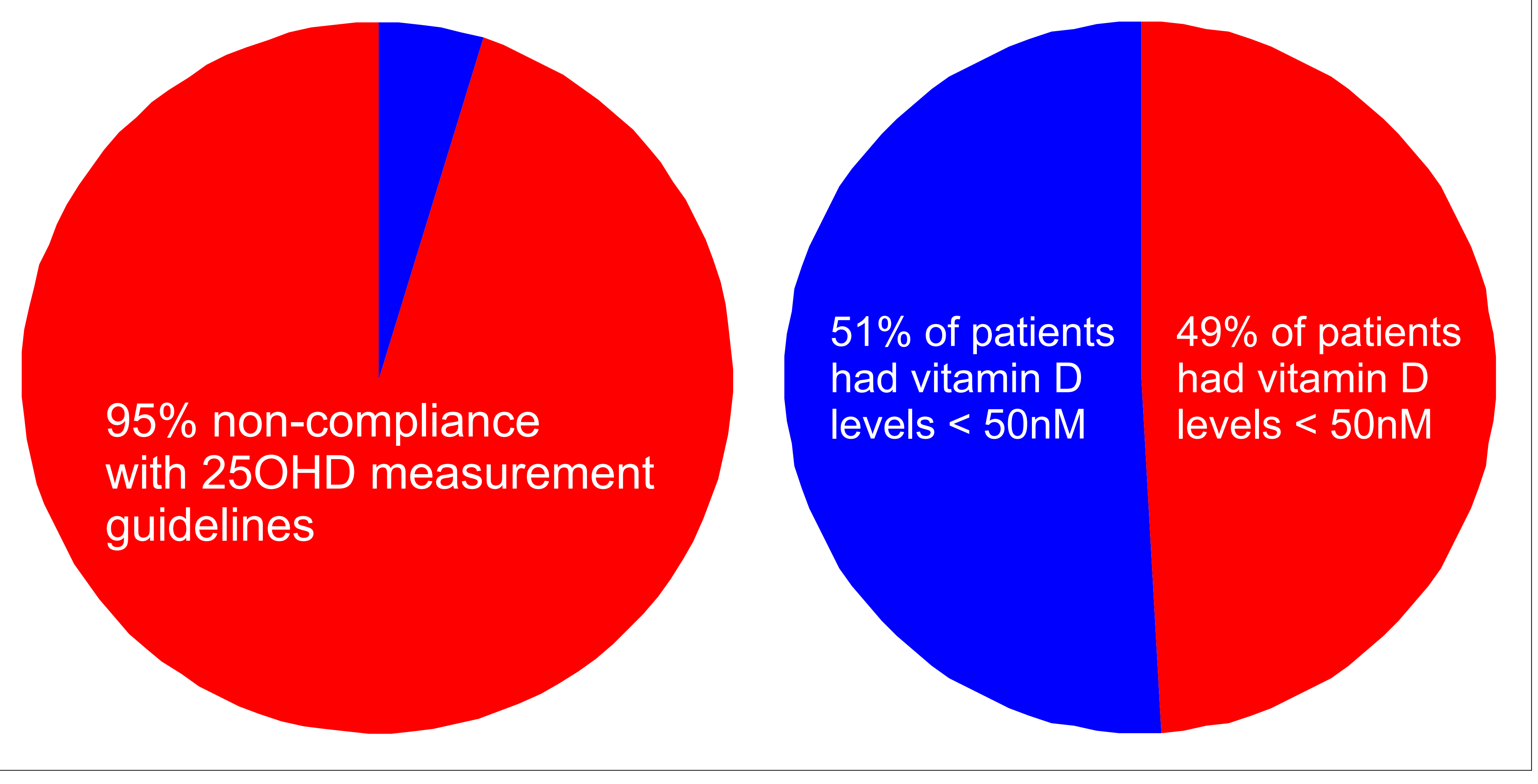

\section{Change effectors}

To optimise vitamin D status and decrease the risk of hypocalcaemia, a three-step approach was taken:

1.Clear written instructions provided to GPs to check serum $25(\mathrm{OH})$ vitamin $\mathrm{D}(25 \mathrm{OHD})$ levels and start oral supplementation (colecalciferol 100,000 units total over 5 days) if $25 \mathrm{OHD}<50 \mathrm{nmol} / \mathrm{L}$.

2.Provision of standard template for use by junior doctors and nurses with clear thresholds for $25 \mathrm{OHD}(>50 \mathrm{nmol} / \mathrm{L})$, corrected calcium $(>2.00 \mathrm{mmol} / \mathrm{L})$ and renal function (eGFR $>40 \mathrm{ml} / \mathrm{min} / 1.73 \mathrm{~m} 2$ for zoledronate)

3. Treatment could only be given if these assessments were completed.

\section{Result of second audit cycle}

After the first audit, a standard of $85 \%$ compliance with guidelines was set as success threshold for the changes put in place. This compliance rate was achieved overall.

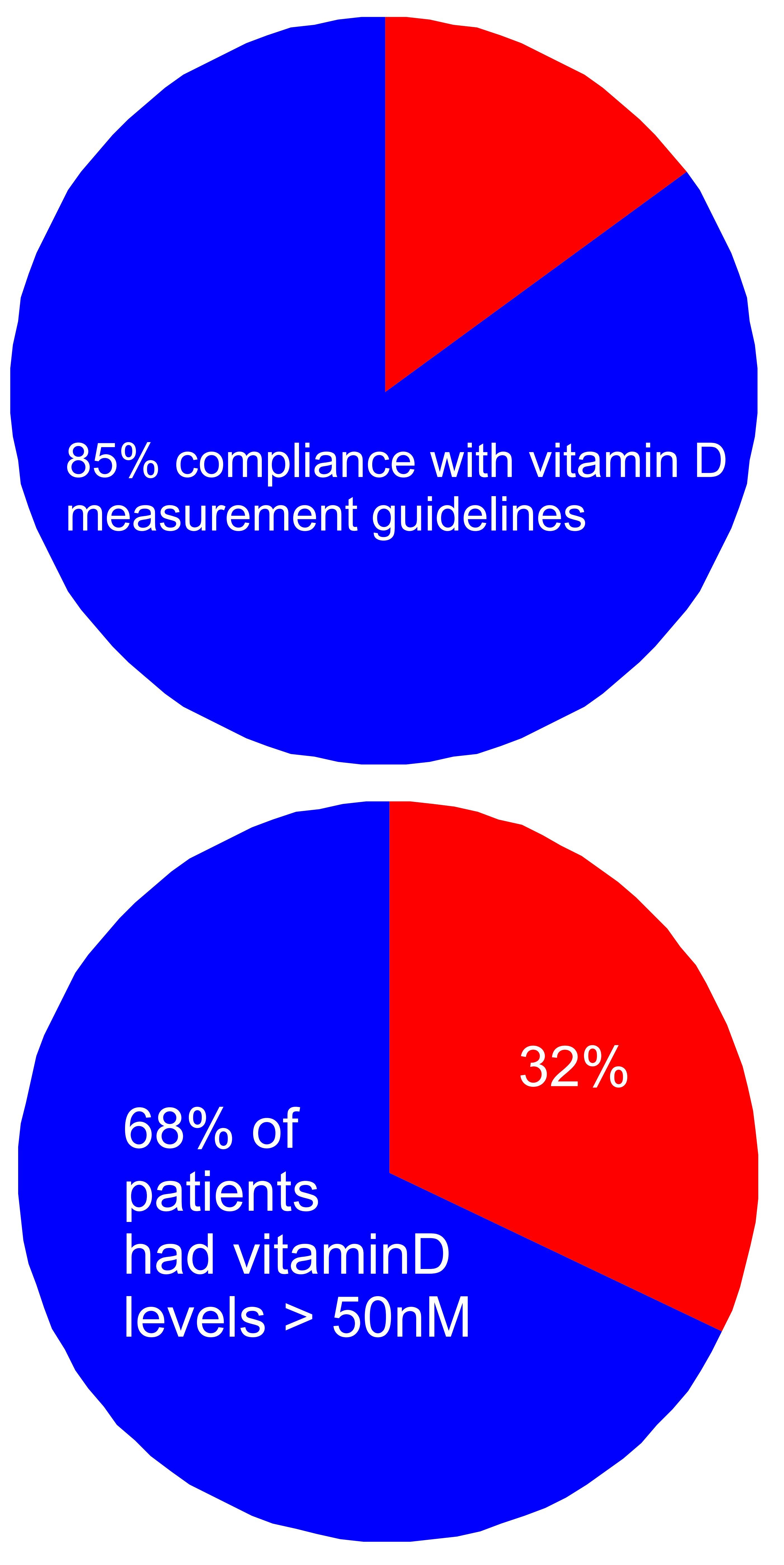

\section{Comparison between audit cycles}

Through the change effectors, we demonstrate an $80 \%$ improvement in the monitoring of $250 \mathrm{HD}$ levels. Importantly, there was a $20 \%$ reduction in the number of patients with very low $(<25 \mathrm{nmol} / \mathrm{L}) 25 \mathrm{OHD}$ levels.

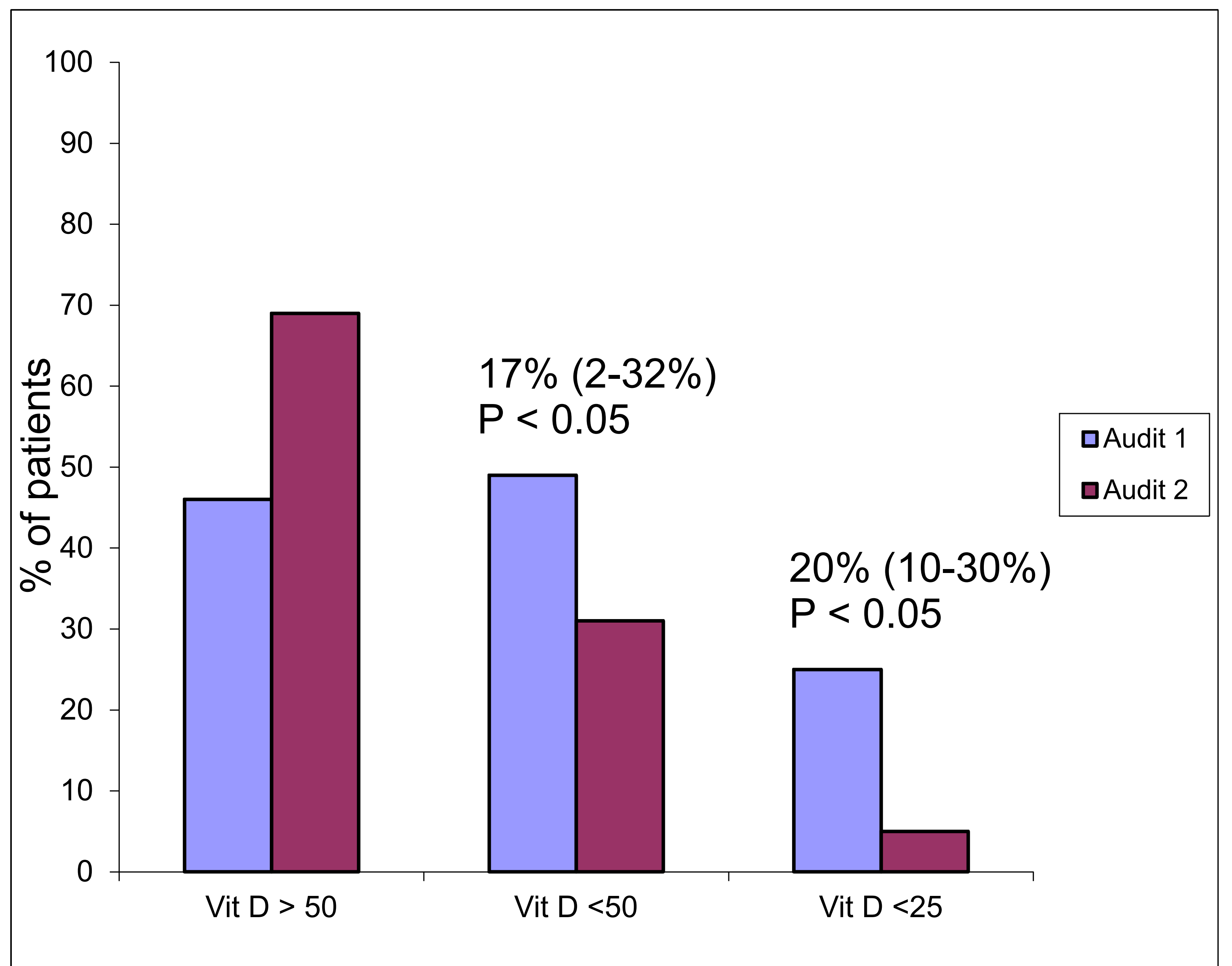

\section{Conclusion}

Vitamin D deficiency was significantly reduced in our patient population, which may have been explained, partially, by season. However, by providing detailed information to GPs and structured templates to hospital junior doctors, we demonstrate a significant improvement in the monitoring of vitamin D levels and appropriate oral vitamin D supplementation in line with current guidance. 